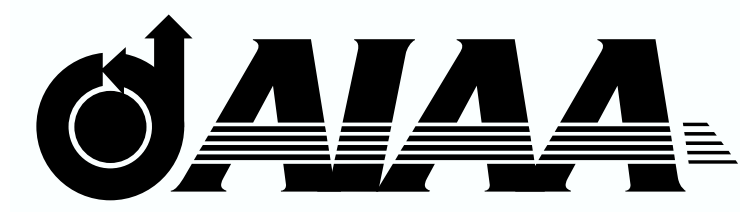

AIAA 2004-2887

\title{
Aeroacoustic Analysis of a Simplified Landing Gear
}

David P. Lockard and Mehdi R. Khorrami NASA Langley Research Center Hampton, VA 23681

Fei Li

High Technology Corporation Hampton, VA

10th AIAA/CEAS Aeroacoustics Conference May 10-13, 2004 Manchester, UK 


\title{
High Resolution Calculation of a Simplified Landing Gear
}

\author{
David P. Lockard, Mehdi R. Khorrami ${ }^{\dagger}$ \\ NASA Langley Research Center \\ Hampton, VA \\ and \\ Fei Li \\ High Technology Corporation \\ Hampton, VA
}

\begin{abstract}
A hybrid approach is used to investigate the noise generated by a simplified landing gear without small scale parts such as hydraulic lines and fasteners. The Ffowes Williams and Hawkings equation is used to predict the noise at far-field observer locations from flow data provided by an unsteady computational fluid dynamics calculation. A simulation with 13 million grid points has been completed, and comparisons are made between calculations with different turbulence models. Results indicate that the turbulence model has a profound effect on the levels and character of the unsteadiness. Flow data on solid surfaces and a set of permeable surfaces surrounding the gear have been collected. Noise predictions using the porous surfaces appear to be contaminated by errors caused by large wake fluctuations passing through the surfaces. However, comparisons between predictions using the solid surfaces with the near-field CFD solution are in good agreement giving confidence in the far-field results.
\end{abstract}

\section{$\underline{\text { Nomenclature }}$}

$\begin{array}{ll}c & \text { speed of sound } \\ f & \text { integration surface defined by } f=0 \\ F_{i} & \text { dipole source terms } \\ H & \text { Heaviside function } \\ i & \sqrt{-1} \\ M_{i} & \text { local source Mach number vector, } v_{i} / c \\ M & \text { Mach number, }\left|M_{i}\right| \\ \hat{n}_{i} & \text { outward directed unit normal vector } \\ p & \text { pressure } \\ \mathbf{Q} & \text { monopole source term } \\ t & \text { time } \\ u_{i} & \text { Cartesian fluid velocity components } \\ v_{i} & \text { Cartesian surface velocity components } \\ x, y, z & \text { Cartesian observer coordinates }\end{array}$

Greek:

$\delta(f) \quad$ Dirac delta function

$\delta_{i j} \quad$ Kronecker delta

$\rho \quad$ fluid density

$\xi, \eta, \zeta \quad$ source coordinates

Superscript:

, perturbation quantity (e.g. $\rho^{\prime}=\rho-\rho_{o}$ )

Subscript:

$o \quad$ freestream quantity

\section{Introduction}

The past thirty years have seen significant reductions in jet noise through the adoption of high-bypass-ratio turbofan engines on civil aviation transports. Formerly unimportant noise sources such as the airframe have now become a major concern for noise certification and environmental considerations. Airframe noise is most important during aircraft approach and

\footnotetext{
*Aerospace Technologist, Senior Member, AIAA

${ }^{\dagger}$ Aerospace Technologist, Associate Fellow, AIAA

This material is declared a work of the U.S. Government and is not subject to copyright protection in the United States.
} 
landing, when engines are operating at reduced thrust with the high-lift devices and landing gear deployed. Wind tunnel tests $^{1-3}$ and fly-over measurements ${ }^{4}$ have revealed the leading-edge slats, flap edges, and the landing gear to be the major contributors to airframe noise. Each of the three primary sources of airframe noise are important on different classes of airplanes, but the main landing gear is a dominant source on most modern wide-body transports. Although flow computations of high-lift devices such as flaps and slats received considerable attention in the last decade, the intricacies of the landing-gear flowfield and its associated sound sources have remained virtually unknown due to overwhelming geometrical complexities. Nonetheless, computational studies of landing gear are beginning to be performed. ${ }^{5}$ Souliez et al. ${ }^{6}$ used an unstructured grid technique to investigate the same baseline landing gear used in this work, but the calculations were restricted to laminar flow, and the geometry was further simplified by removing the door. In this paper, we present an analysis of our computational aeroacoustic study of a model landing gear. Our computational approach involves a hybrid strategy. In the first step we perform an unsteady computational fluid dynamics (CFD) simulation to provide a highly resolved near-field solution. Two CFD computations have been performed to investigate the influence of the turbulence model on the unsteady flow. Unsteady Reynolds averaged Navier-Stokes (URANS) and detached eddy simulations ${ }^{7}$ (DES) have been performed. The results indicate that the turbulence model can greatly influence the levels and character of the predicted noise.

Despite continued advances in computational resources and numerical algorithms, it is still prohibitively expensive and often infeasible to attempt to resolve wave propagation from near-field sources to far-field observers. Integral techniques that can predict the far-field signal based solely on near-field input are a means to overcome this difficulty. Hence, the Ffowcs Williams-Hawkings (FW-H) equation ${ }^{8}$ solver described by Lockard ${ }^{9}$ is used to predict the acoustic signature at various observer locations using unsteady flow data from the CFD calculation.

\section{Acoustic Equations}

The FW-H equation can be written in differential form ${ }^{10}$ as

$$
\left(\frac{\partial^{2}}{\partial t^{2}}-c_{o}^{2} \frac{\partial^{2}}{\partial x_{i} \partial x_{i}}\right)\left(H(f) \rho^{\prime}\right)=\frac{\partial^{2}}{\partial x_{i} \partial x_{j}}\left(T_{i j} H(f)\right)-\frac{\partial}{\partial x_{i}}\left(F_{i} \delta(f)\right)+\frac{\partial}{\partial t}(Q \delta(f))
$$

where

$$
\begin{aligned}
T_{i j} & =\rho u_{i} u_{j}+P_{i j}-c_{o}^{2} \rho^{\prime} \delta_{i j}, \quad F_{i}=\left(P_{i j}+\rho u_{i}\left(u_{j}-v_{j}\right)\right) \frac{\partial f}{\partial x_{j}}, \text { and } \\
Q & =\left(\rho_{o} v_{i}+\rho\left(u_{i}-v_{i}\right)\right) \frac{\partial f}{\partial x_{i}} .
\end{aligned}
$$

The dipole term $F_{i}$ involves an unsteady force, and $Q$ gives rise to a monopole-type contribution that can be thought of as an unsteady mass addition. The function $f=0$ defines the surface outside of which the solution is desired. The normalization $|\nabla f|=1$ is used for $f$. The total density and pressure are given by $\rho$ and $p$, respectively. The fluid velocities are $u_{i}$, while the $v_{i}$ represent the velocities of the surface $f$. The Kronecker delta, $\delta_{i j}$, is unity for $i=j$ and zero otherwise. The ambient speed of sound is denoted by $c_{o}$. A prime is used to denote a perturbation quantity relative to the free-stream conditions denoted by the subscript $o$. The Cartesian coordinates and time are $x_{i}$ and $t$, respectively. The usual convention, which is followed here, involves a quiescent ambient state with $f$ prescribed as a function of time so that it always surrounds a moving source region of interest. $H(f)$ is the Heaviside function which is unity for $f>0$ and zero for $f<0$. The derivative of the Heaviside function $H^{\prime}(f)=\delta(f)$ is the Dirac delta function, which is zero for $f \neq 0$, but yields a finite value when integrated over a region including $f=0$. The inviscid part, $P_{i j}=p \delta_{i j}$, of the compressive stress tensor $P_{i j}$ is used in this work.

The FW-H equation is an exact rearrangement of the Navier-Stokes equations that allows one to determine the acoustic signal at distant observer locations if the details of the source region are already known. Hence, the Navier-Stokes equations still need to be solved, but only where nonlinear and viscous effects are important. All of the linear propagation can be determined by the FW-H equation. For three-dimensional flows, the time-domain FW-H formulations developed by Farassat ${ }^{11}$ are efficient and amenable to numerical computations. Some additional efficiency can be obtained by restricting the source to uniform, rectilinear motion. Furthermore, the equation can be solved in the frequency domain which can be useful if one is only interested in analyzing certain frequencies. The frequency domain solution of FW-H equation can be written in the form ${ }^{9}$

$$
H(f) c_{o}^{2} \rho^{\prime}(\mathbf{y}, \omega)=-\int_{f=0} F_{i}(\boldsymbol{\xi}, \omega) \frac{\partial G(\mathbf{y} ; \boldsymbol{\xi})}{\partial y_{i}} d s-\int_{f=0} i \omega Q_{n}(\boldsymbol{\xi}, \omega) G(\mathbf{y} ; \boldsymbol{\xi}) d s+I_{Q}
$$


where

$$
F_{i}=\left(p \delta_{i j}+\rho\left(u_{i}-U_{i}\right)\left(u_{j}+U_{j}\right)+\rho_{o} U_{i} U_{j}\right) \hat{n}_{j}, \quad Q_{n}=\left(\rho\left(u_{i}+U_{i}\right)-\rho_{o} U_{i}\right) \hat{n}_{i} .
$$

The volumetric quadrupole term is denoted by $I_{Q}$ and includes effects such as nonlinear propagation and refraction. In this work, the volumetric contribution is expected to be small and is neglected. Souliez et al. ${ }^{6}$ performed FW-H predictions of landing gear noise using solid and permeable integration surfaces and found the solutions to be nearly identical in the far-field, although discrepancies were noted in the near field. The porous surface enclosed a significant region around the gear and should have included most of the flow interaction effects. In this work, computations with solid and porous integration surfaces are performed. Computations with the porous surfaces appear to be contaminated because of strong wakes passing through the surfaces. However, the calculations with the solid surfaces are in good agreement with CFD data in the flow field as long as wakes are not present.

\section{Simplified Landing Gear Model}



(a) Surface grid

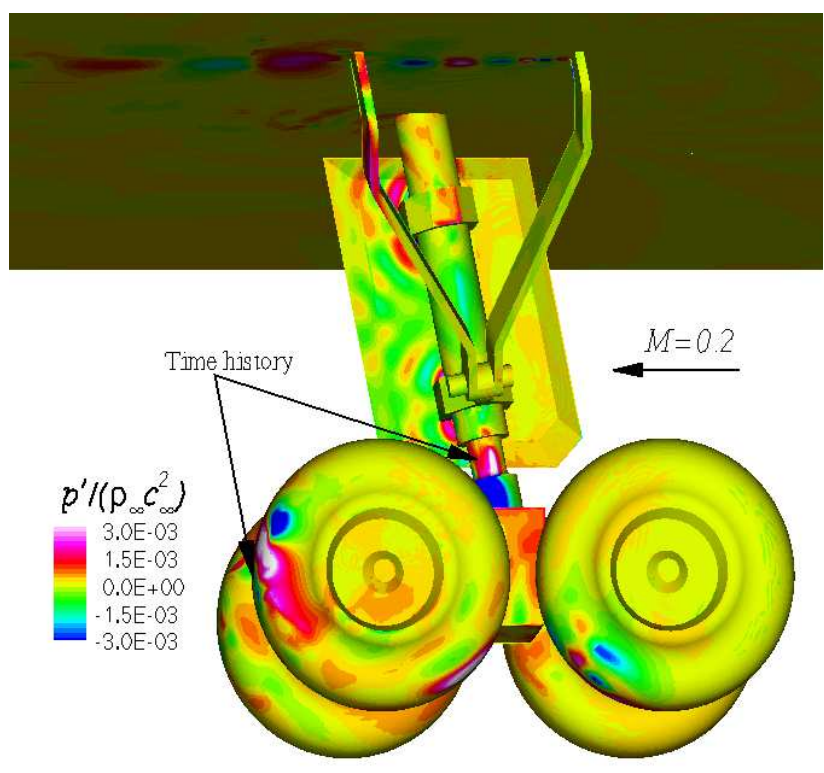

(b) Perturbation pressure on solid surfaces

Fig. 1 CFD grid and SST results for a landing gear.

The simulated geometry is a four-wheel landing gear model that approximately represents a ten percent scale Boeing 757 main landing gear. The model geometry is fairly complex and composed of four wheels, two side struts, an oleo, a side-door, yokes, a pin, and other structures that join the system together (figure 1 (a)). The gear assembly is mounted on a flat plate that represents the aircraft wing. The structured grid consists of 155 blocks possessing a total of 13.3 million grid points. In reference 12 the authors discussed the results for a CFD grid of half the resolution of the current calculation. Figure 1(a) also shows the grid distribution on the surface of the landing gear. The reference length scale is the gear wheel diameter $(3.7 \mathrm{in}=0.09398 \mathrm{~m}$ ) and the freestream Mach number is 0.2 . The CFD calculation employs the three-dimensional, time-dependent code CFL3D, ${ }^{13,14}$ developed at NASA Langley Research Center to solve the threedimensional, time-dependent, thin-layer Reynolds-Averaged Navier-Stokes (RANS) equations. A more detailed discussion of the CFD calculation can be found in the paper by Li et al. ${ }^{15}$ We have used an unsteady RANS calculation with the shear stress transport (SST) $k-\omega$ turbulence model of Menter ${ }^{16,17}$ which was developed for steady flow. Although qualitatively good results have been obtained with this approach for unsteady problems, ${ }^{18,19}$ it is well known ${ }^{19}$ that the turbulence model is overly dissipative. We have also run the landing gear problem as a detached-eddy simulation ${ }^{7}$ (DES) as proposed by Spalart. ${ }^{20,21}$ The DES model essentially reduces the level of eddy viscosity in regions away from solid surfaces when the grid is sufficiently fine.

Contours of the instantaneous perturbation pressure fluctuations on the gear solid surfaces are displayed in figure 1(b). The surface pressure shows the footprint of the highly nonlinear and complicated interactive near-field flow dynamics. Lazos $^{22,23}$ investigated a simpler four wheel landing gear experimentally and also found the flow around the wheels to be quite complicated. Figure 2(a) compares the SST and DES time histories of the pressure on the downstream wheel opposite the door as indicated by the arrow in figure 1(b). The pressure is nondimensionalized by $\rho_{o} c_{o}^{2}$. The time histories show the irregular character of the signal. The corresponding spectra are shown in figure 2(b). The narrow band results have been 
normalized to $1 \mathrm{~Hz}$ bin widths. The DES solution has considerably higher fluctuation levels at all frequencies. The high frequency oscillations above $20 \mathrm{kHz}$ are generated by resonances in small, triangular shaped spaces between the yokes and the door. The wave pattern seen on the door in figure 1(b) is caused by these tones. In the SST calculation, the phenomenon results in tones around 21 and $26 \mathrm{KHz}$, but only the $26 \mathrm{kHz}$ tone is observable at this location. Several tones and a broad increase above $20 \mathrm{kHz}$ are evident in the DES solution. Figure 3 compares the solutions on the oleo in the contraction just below the door. The SST solution is more regular at this location, but the overall amplitude of the fluctuations is similar.



(a) Time history



(b) spectra

Fig. 2 Pressure signal on the downstream wheel opposite the door.



(a) Time history



(b) Spectra

Fig. 3 Pressure signal on the oleo.

Figures 4 and 5 present the mean pressure coefficient and RMS pressure levels on the gear surfaces from the two CFD calculations. The mean pressure is fairly similar, although the suction peak on the oleo is stronger in the DES calculation. Furthermore, the pressure distribution on downstream side of the rear tire is somewhat altered. Considerably more discrepancies can be observed in the RMS pressure contours. The regions with high fluctuation levels in the SST calculation are also prominent in the DES results, but the levels are noticeably higher for the DES. In addition, the area over which high fluctuation levels are present is much larger for the DES calculation. Although the RMS pressure contours give an indication of the total fluctuation energy in the flow, they do not convey any information about the scales of the fluctuations. Figure 6 presents a comparison of the vorticity magnitude in a plane just downstream of the rear wheels. The vorticity levels in the SST computation are considerably lower, and the wakes emanating from different sides of components remain distinct. In the DES calculation, all the wakes have merged, and the region is filled with vorticity 
of various scales. Considerably more interaction appears to be occurring between the vortical structures and indicates increased energy transfer between scales. The higher levels in the spectra in figures 2(b) and 3(b) also suggest that more energy is being transferred into high frequencies without being excessively dissipated. The comparison between the DES and SST solutions indicates that the DES calculation is more consistent with the anticipated physics, but such a comparison is not able to verify that the DES calculation is correctly capturing all of the nonlinear energy transfer.

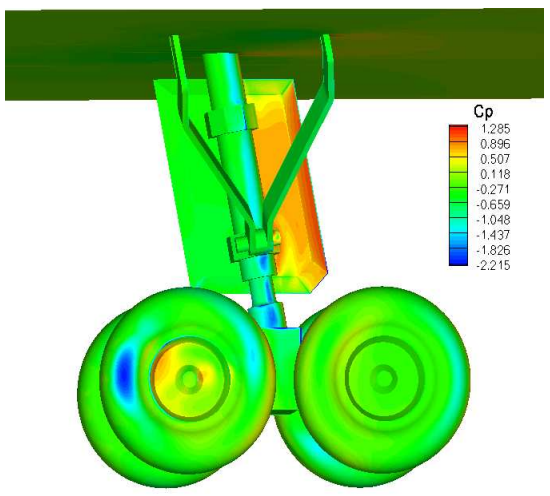

(a) $\mathrm{Cp}$

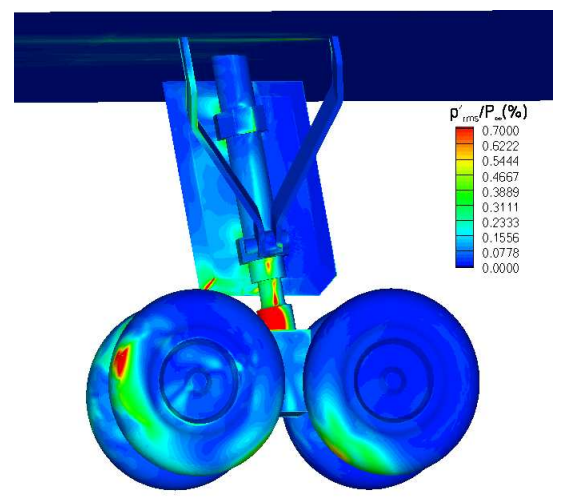

(b) RMS pressure



(c) RMS pressure

Fig. 4 SST mean and perturbation results for a landing gear. The Flow is from right to left.

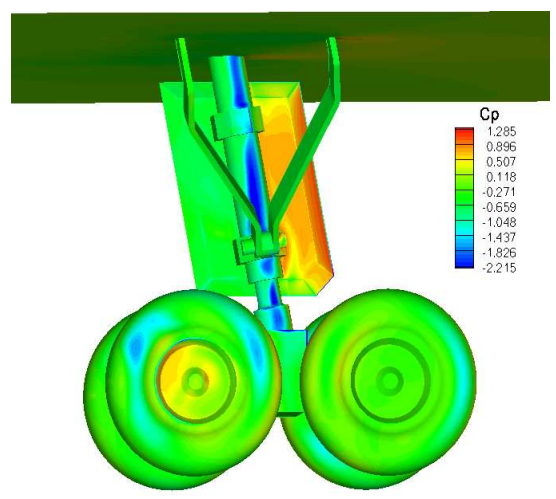

(a) $\mathrm{Cp}$

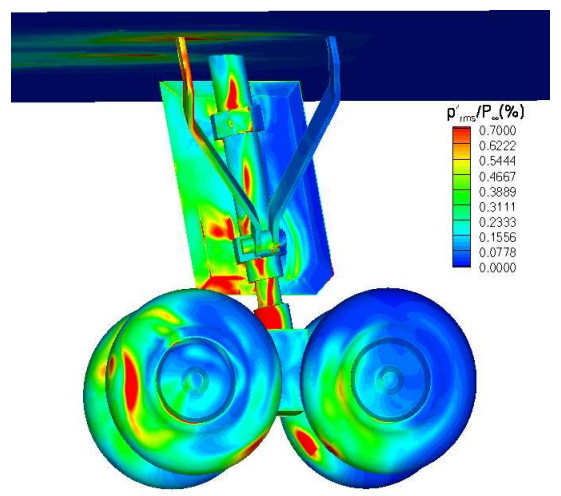

(b) RMS pressure

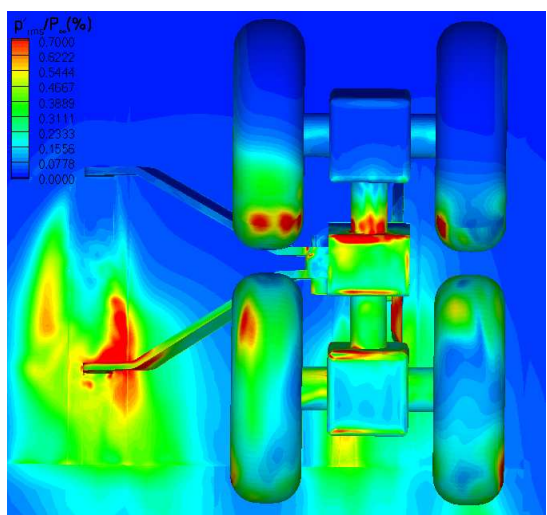

(c) RMS pressure

Fig. 5 DES mean and perturbation results for a landing gear. The Flow is from right to left.



(a) SST

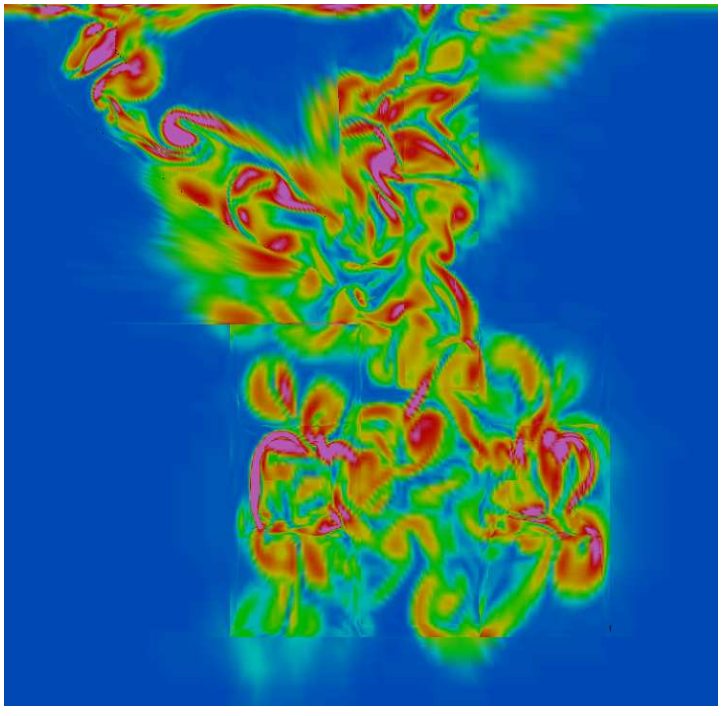

(b) DES

Fig. 6 Vorticity magnitude in a cross-stream plane downstream of the landing gear. The Flow is out of the page. 


\section{Impenetrable Surface Noise Calculations}

The noise calculations involve 181 total subsurfaces comprising the impermeable data surface. Only the pressure is need on impenetrable surfaces. 147 subsurfaces are on the gear itself, and 34 are on the plate above the gear. The subsurfaces are a natural consequence of the block structured grid used for the CFD calculation. Each subsurface is a boundary of one of the 155 blocks comprising the grid.

Over 12,000 nondimensional time samples with $l / c_{o} \Delta t=0.02$ have been collected from the SST calculation. Nearly 7, 000 samples have been collected from the DES simulation. The computations are sampled at every fourth time step. A Ffowcs Williams-Hawkings solver ${ }^{9}$ written specifically for airframe noise applications is being used to perform the noise calculations. The observer is located 100 wheel diameters away from the gear. All of the results in the paper represent an average over five time histories with 50\% overlap. Each segment consists of 4096 samples for the SST calculations; whereas, 2048 are used for the DES cases.

\section{Subsurface Noise Predictions}

Although much of the smaller scale detail is missing from this gear model, it is not apparent which components are contributing to the different portions of the spectrum. To investigate the dominant sources in each frequency range, the landing gear was divided into 10 regions as shown in figure 7(a). Each region is colored differently to identify each of the subdomains.

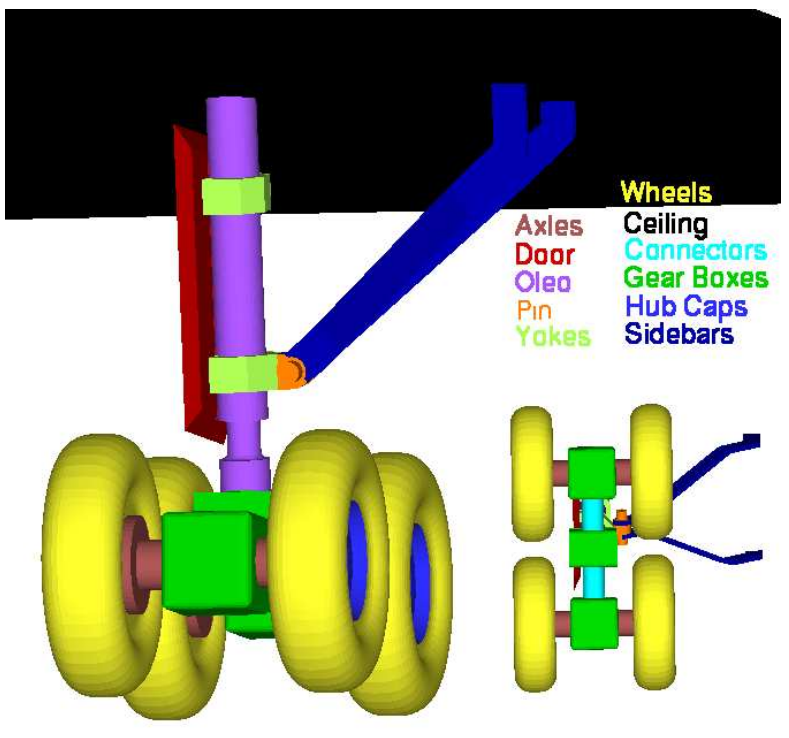

(a) Subsurfaces



(b) Pressure contours

Fig. 7 Landing gear subsurfaces colored by component and instantaneous pressure contours from SST calculation.

The prediction using all of the surfaces (Gear + Ceiling) is compared with the results when using the ceiling, and everything except the ceiling (No Ceiling) in figure 8 . The presented results are from the frequency-domain solver, although calculations in the time-domain show good agreement. The results are for an observer located 100 wheel diameters directly below the gear. The results do vary with observer position, but the general trends are similar at most observer locations of interest. However, most of the noise from the ceiling is associated with extraneous noise sources in the vicinity of patched regions of the grid. Because of the extreme complexity of the geometry, extensive patching is used to prevent the propagation of fine resolution to unimportant regions and thus reduce the number of grid points. Unfortunately, the disparity in grid resolution across interfaces is often insufficient to resolve the phenomena trying to pass through the boundaries resulting in numerical oscillations.

Figure 7(b) shows the instantaneous perturbation pressure contours on the gear and ceiling looking from below. The most intense fluctuations occur in the wake of the sidebars. Although some interaction between unsteadiness in the wakes with the ceiling is expected, the pressure fluctuations on the ceiling have been amplified by numerical instabilities in the regions around patched interfaces. Both the SST and DES noise calculations are contaminated by these numerical errors when the ceiling is included. Useful information about the radiated noise can still be ascertained by examining the noise radiated from individual components.

The predicted noise for each of the subsurfaces in figure 7 is shown in figures 9(a)-(1). Note that the horizontal axes for 9(a)-(b) are different from the rest of the figures so that the effect of the high frequency oscillations in the cavities between the yokes and the door can be seen. Just as the surface spectra in figure 2(b) showed a broad increase above $20 \mathrm{kHz}$ with several tones for the DES calculation, the noise prediction shows similar trends. In general, the SST results are more tonal, 


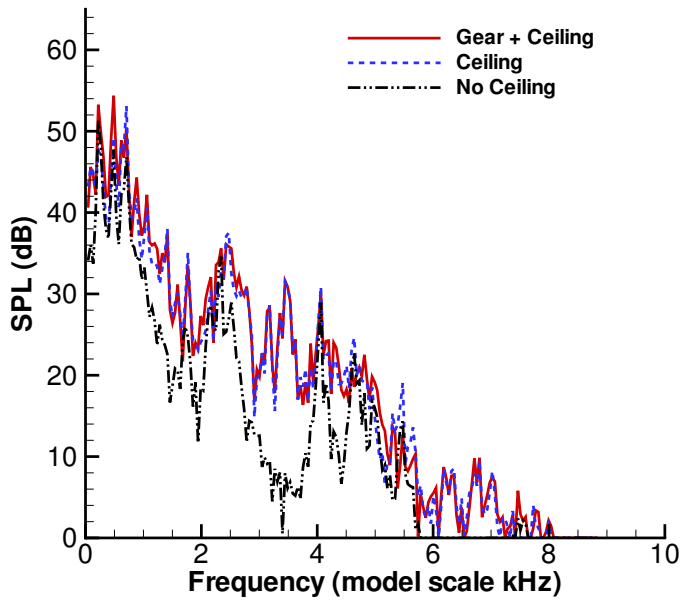

(a) SST

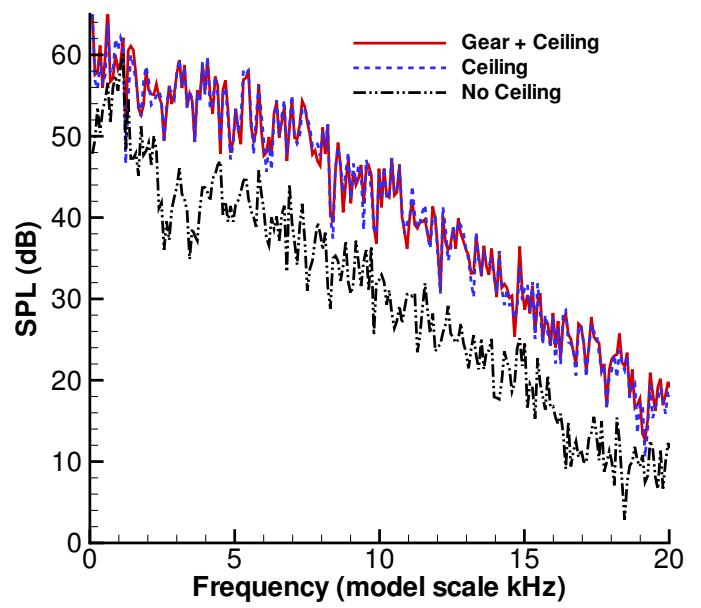

(b) DES

Fig. 8 Subsurface spectra for an observer 100 wheel diameters below the landing gear.

and the decay rate with frequency is much more rapid. However, the peak frequency for many of the components is similar between the two computations. Furthermore, the wheels and gear boxes appear to be responsible for most of the noise in both calculations. In addition, both computations indicate that the sidebars primary contribution is a tone near $1 \mathrm{kHz}$.

In all cases, the DES noise predictions have much higher levels, and the difference becomes greater as the frequency increases. Although experimental measurements of the noise from the simplified gear is currently unavailable, comparisons with measurements from a 6 wheeled, $26 \%$, Boeing 777 landing-gear model ${ }^{24}$ revealed that the DES results are much closer to the measurements, although the fall off with frequency is still slightly too rapid. The similarity between the shapes of the spectra from different components in the DES calculation indicates that at least a portion of the noise is caused by the interactions between the global, turbulent flow with the solid components. The level of turbulence in the flow is more accurately represented in the DES calculation, although there is still some extra diffusion in the computation that causes the decay with frequency to be too rapid. The lack of small-scale parts in the model also contributes to discrepancies at high frequencies.

\section{FW-H Source Strengths}

Another method for identifying source locations involves plotting the integrand in equation 3 over the entire integration surface to visually show where the noise is being generated. For the frequency domain approach, this can be done for each frequency of interest. The amplitude of the complex integrand gives an indication of potential noise sources. One can be deceived by the results because the phase information is lost when only the amplitude of the complex integrand is interrogated. By examining the real and imaginary components, one can get an idea of whether the signals will combine constructively or destructively.

Figures 10 and 11 present the source strengths at three frequencies for the SST and DES calculations. The observer is located 100 wheel diameters directly below the gear. The primary view is at a slight angle from under the gear, and the superimposed image is a view from the side. The amplitudes are dimensionless, and should only be used to make relative comparisons. In general, the levels are considerably higher and more of the gear sees significant source levels for the DES case. Although the ceiling appears to be an important source, it is impossible to discern how strong the real sources are because of the errors in the CFD around patched interfaces on the ceiling.

In general, the gear boxes appear to be the most important source, especially at the higher frequencies. However, figures 9(c) and (d) show that the contribution from the wheels is slightly higher than for the gear boxes, even out to high frequencies. Part of the reason for this is the greater surface area of the wheels, but the phase of the complex source strength is actually a more important factor. The real and imaginary parts of the FW-H integrand switch signs repeatedly over the gear boxes which results in cancellation when integrated over the area. The real and imaginary parts do not oscillate as significantly on the wheels. Hence, there appears to be greater coherence on the wheels. Whether this would be true for real wheels with grooves is unclear.

\section{Directivity}

The directivities from the gear components excluding the ceiling are presented in figure 12. Beyond the disparity in levels between the results from the two calculations, the DES predictions show much greater variation. The primary noise radiation is behind the gear in the DES results. However, somewhat higher levels are also observed to the sides of the gear, 


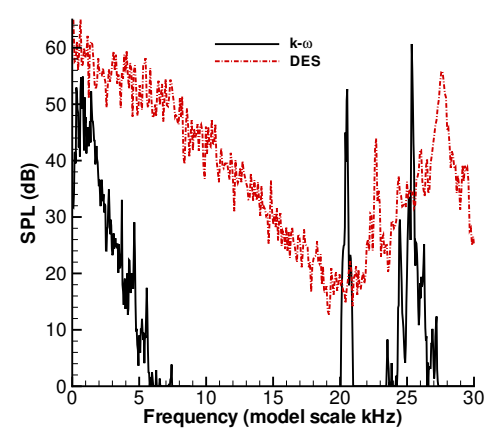

(a) All components (including ceiling)

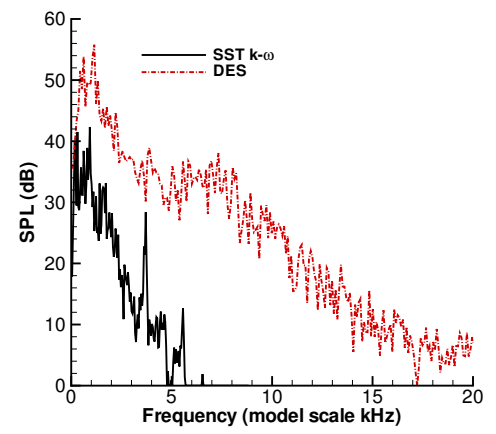

(d) Gear boxes

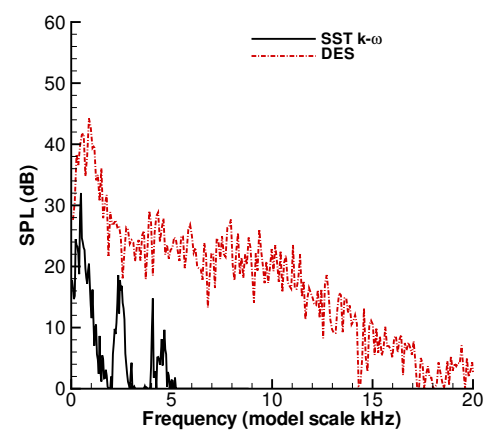

(g) Axles

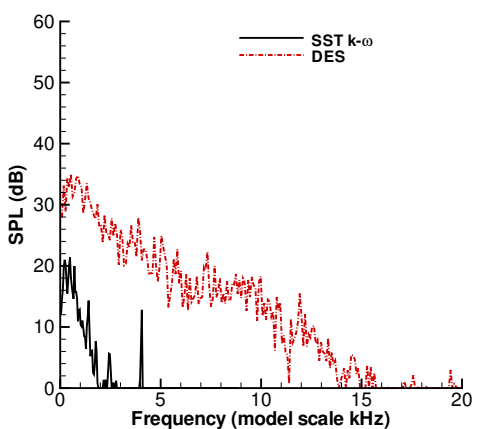

(j) Door



(b) All components (excluding ceiling)



(e) Gear box connectors

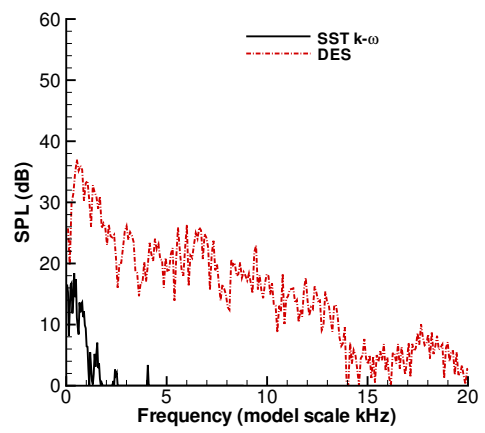

(h) Yokes



(k) Pin

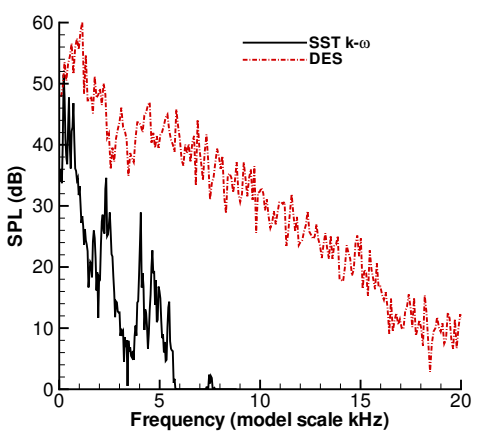

(c) Wheels

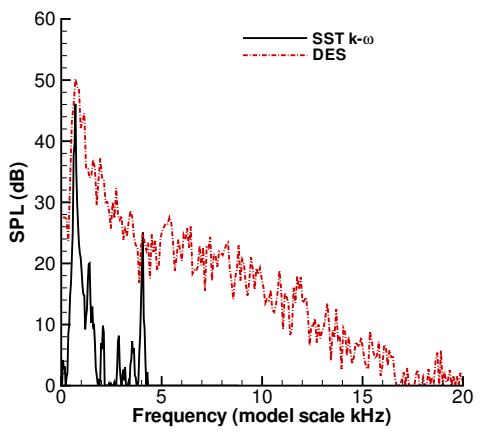

(f) Sidebars



(i) Oleo



(l) Hub caps

Fig. 9 FW-H results for an observer 100 wheel diameters directly below the landing gear. 


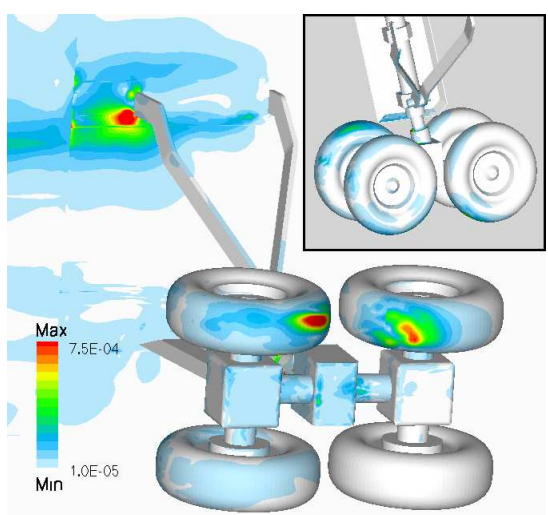

(a) $353 \mathrm{~Hz}$

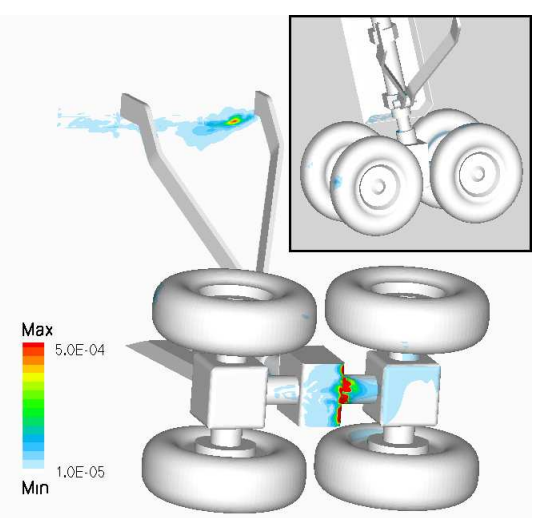

(b) $4637 \mathrm{~Hz}$



(c) $10,000 \mathrm{~Hz}$

Fig. 10 FW-H source strengths from SST calculation. The Flow is from right to left.

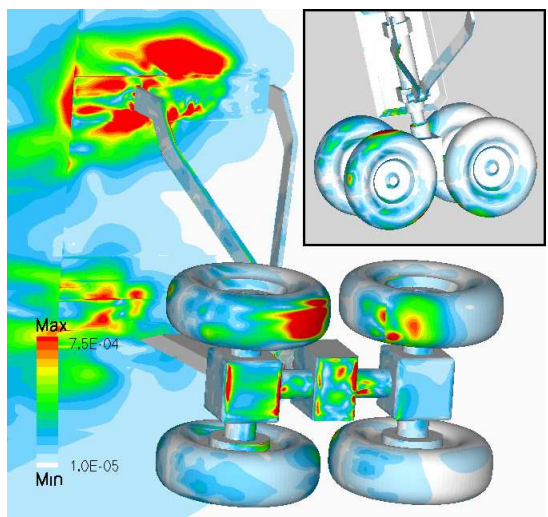

(a) $353 \mathrm{~Hz}$



(b) $4637 \mathrm{~Hz}$

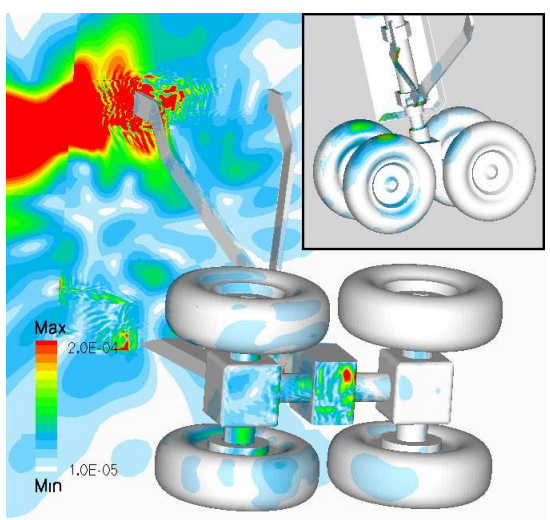

(c) $10,000 \mathrm{~Hz}$

Fig. 11 FW-H source strengths from DES calculation. The Flow is from right to left.

with the region opposite the door being most prominent. The minimum is nearly directly below the gear. For the SST computation, the primary noise radiation is to the sides of the gear, especially opposite the door. In complete contrast to the DES calculation, the minimum occurs behind the gear. Clearly, the differences in the directivities involve more than just changes in fluctuation levels.

\section{Porous Surface Predictions}

Unsteady flow data has been collected on the permeable surfaces surrounding the landing gear as shown in figure 13(a). These surfaces were placed relatively close to the gear so they would be within regions of fine resolution. Unfortunately, some of the surfaces are subjected to strong wakes. Figure 13(b) shows RMS streamwise velocity contours normalized by the freestream velocity. The perturbation fluctuation levels exceed $25 \%$ of the mean around the wheels and behind the oleo. Significant velocity fluctuations are also present around the sidebars. Although the FW-H equation has been used successfully for cylinder shedding problems ${ }^{9,25}$ when wakes encounter porous surfaces, errors are generated as the vortices pass through because the volumetric contribution, which is neglected, should cancel some of the surface terms that are included. When the strength of the vortices is sufficiently large, errors can be generated that are of the same order of the radiated noise.

FW-H predictions of the noise using the permeable surfaces produces levels that are significantly higher than those when using the solid surfaces. Because the errors caused by vortices passing through the surfaces were suspected as the cause, calculations were made for an observer just off of the upstream panel in front of the wheels in figure 13(a). The calculated signals are compared with the density from the CFD computation in figure 14. Clearly, the calculation using the solid surfaces is in much better agreement with the CFD. The dominant frequencies in the porous solution computation actually correspond to the frequencies of the shedding around the wheels and sidebars. Attempts to exclude portions of porous surfaces with the largest wake fluctuations have not yielded reasonable results. Although our porous surface data is useful to query the state of the flowfield, it is not appropriate for making noise predictions with the FW-H solver.

\section{Conclusions}

The current calculations show that numerical simulations of the noise from landing-gear are possible, but they are far too computationally intensive to be used as a design tool. Using a cluster of forty $2.53 \mathrm{GHz}$ Intel P4 computers with a 


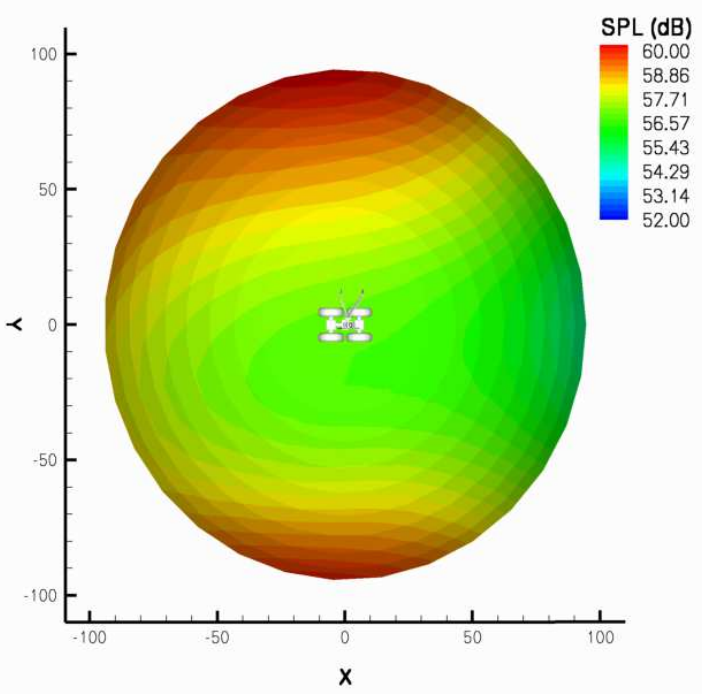

(a) SST

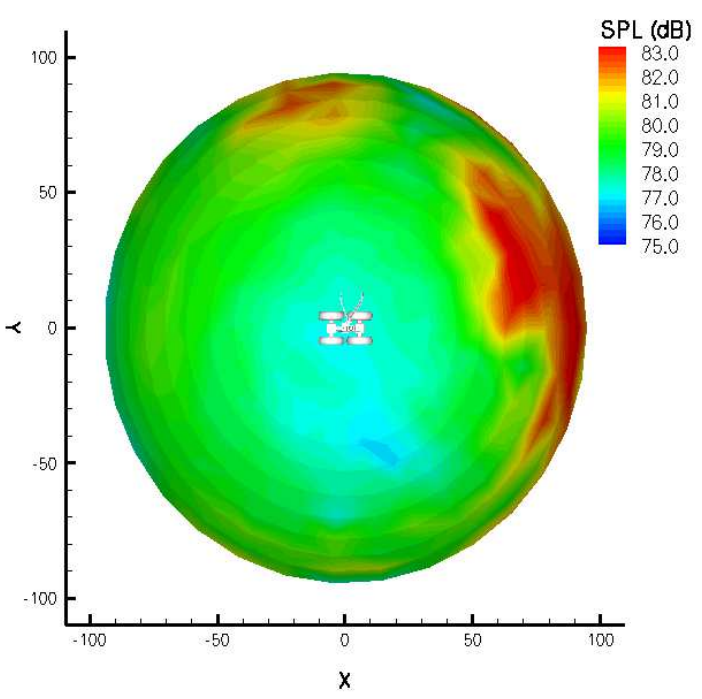

(b) DES

Fig. 12 Directivity contours for an observer located on a hemisphere of radius 100 wheel diameters from the landing gear. The flow is from left to right.

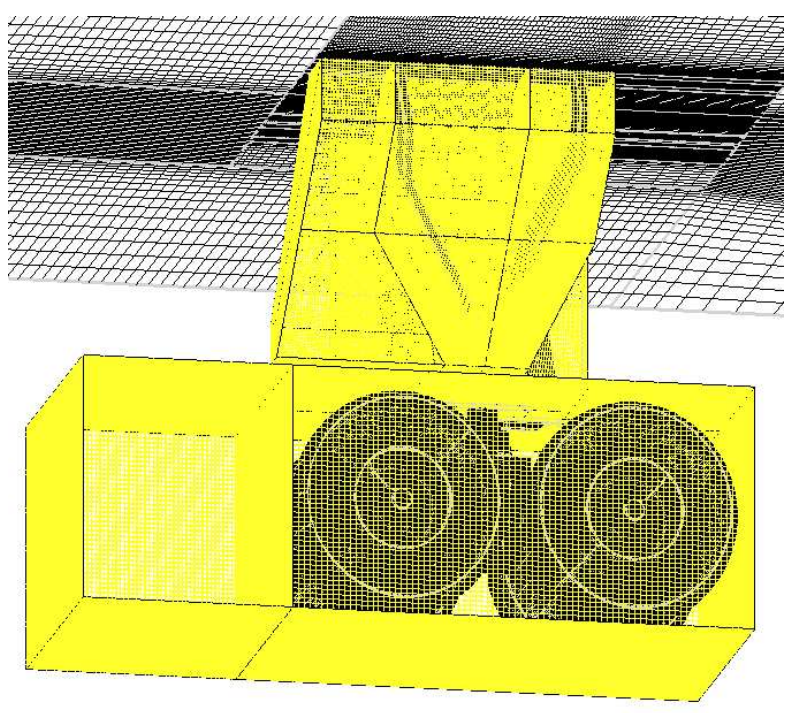

(a) Surfaces

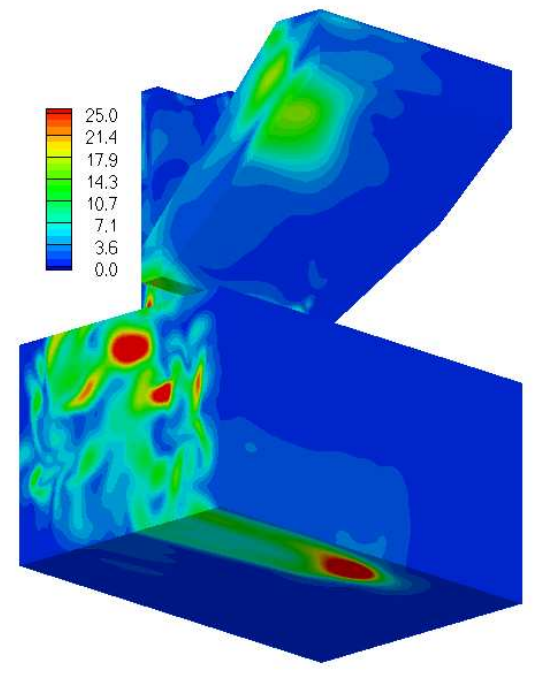

(b) RMS $u / U_{\infty} \%$

Fig. 13 Penetrable surfaces used for data collection and RMS streamwise velocity from the SST computation. The Flow is from right to left.

high-speed Myrinet network, nearly 6 months would be required just to accumulate a data record of the size used in this work. Nonetheless, the data is extremely useful to guide the development of simple models. However, the differences between the SST and DES results suggests that further validation is needed. Experimental measurements are planned to verify some of the current results. We are also pursuing advances in numerical algorithms, grid generation, and turbulence modeling that should improve our simulation capability.

In the DES calculation, most of the gear is engulfed in regions of high turbulence that tends to minimize regular vortex shedding. Geometric irregularities also seem to prevent regular shedding. The peaks in the spectra still correspond reasonably well with those that would be predicted based on Strouhal shedding, but the spectral shapes are very broad rather than exhibiting distinct tones. The sidebars are an exception, perhaps because of their rectangular shape, orientation, and distance from most of the other gear components. Despite the complexity of the problem, a picture is emerging of the characteristic flowfield around a landing gear that will be used to guide future modeling efforts.

\section{References}

${ }^{1}$ Choudhari, M. M., Lockard, D. P., Macaraeg, M. G., Singer, B. A., Streett, C. L., Neubert, G. R., Stoker, R. W., Underbrink, J. R., Berkman, M. E., Khorrami, M. R., and Sadowski, S. S., "Aeroacoustic Experiments in the Langley Low-Turbulence Pressure Tunnel," NASA TM $2002-211432$. 


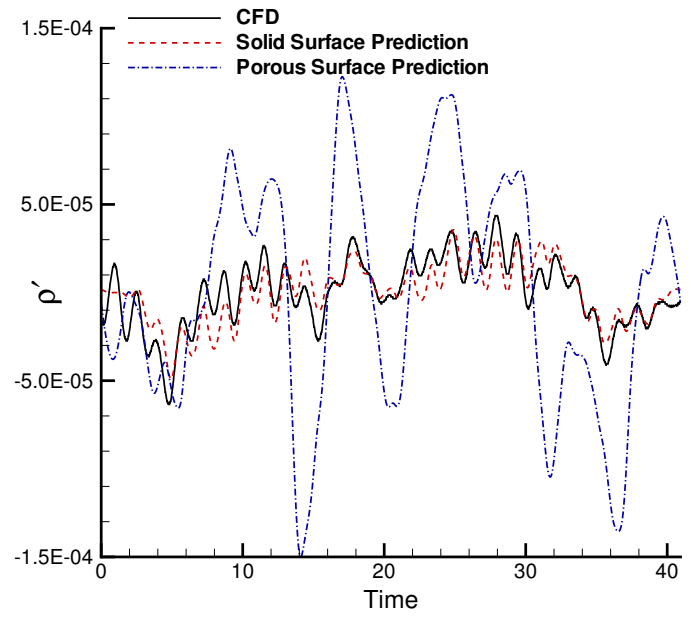

(a) Time history

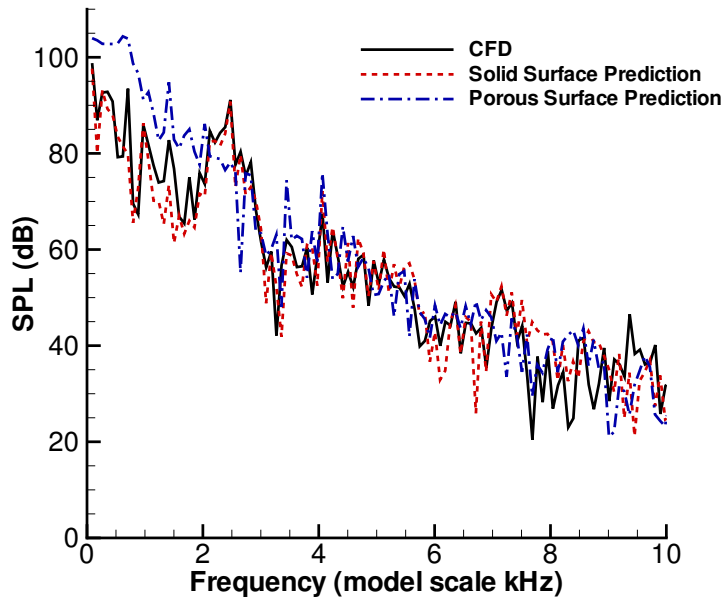

(b) Spectra

Fig. 14 Comparison of CFD and FW-H predictions for an observer near the upstream porous surface in front of the wheels.

${ }^{2}$ Streett, C. L., Lockard, D. P., Singer, B. A., Khorrami, M. R., and Choudhari, M., "In Search of the Physics - The Interplay of Experiment and Computation in Airframe Noise Research; Flap-edge Noise," AIAA-03-0979, 41st AIAA Aerospace Sciences Meeting \& Exhibit, Reno, NV, 2003.

${ }^{3}$ Khorrami, M. R., Choudhari, M., Singer, B. A., Lockard, D. P., and Streett, C. L., "In Search of the Physics - The Interplay of Experiment and Computation in Slat Aeroacoustics," AIAA-03-0980, 41st AIAA Aerospace Sciences Meeting \& Exhibit, Reno, NV, 2003.

${ }^{4}$ Stoker, R., Guo, Y., Streett, C., and Burnside, N., "Airframe Noise Source Locations of a 777 Aircraft in Flight and Comparisons with Past Model-Scale Tests," AIAA-03-3111, Presented at the 9th AIAA/CEAS Aeroacoustics Conference and Exhibit in Hilton Head, SC, 2003.

${ }^{5}$ Hedges, L. S., Travin, A. K., and Spalart, P. R., "Detached-Eddy Simulations Over a Simplified Landing Gear," Journal of Fluids Engineering, Vol. 124, 2002, pp. 413-423.

${ }^{6}$ Souliez, F. J., Long, L. N., Morris, P. J., and Sharma, A., "Landing Gear Aerodynamic Noise Prediction Using Unstructured Grids," International Journal of Aeroacoustics, Vol. 1, No. 2, 2002, pp. 115-135.

${ }^{7}$ Squires, K. D., Forsythe, J. R., Morton, S. A., Strang, W. Z., Wurtzler, K. E., Tomaro, R. F., Grismer, M. J., and Spalart, P. R., "Progress on Detached-Eddy Simulation of Massively Separated Flows," AIAA-02-1021, Presented at the 40th AIAA Aerospace Sciences Meeting and Exhibit in Reno, NV, 2002.

${ }^{8}$ Ffowcs Williams, J. E. and Hawkings, D. L., "Sound generation by turbulence and surfaces in arbitrary motion," Philosophical Transactions of the Royal Society of London A, Vol. 342, 1969, pp. 264-321.

${ }^{9}$ Lockard, D. P., "A Comparison of Ffowcs Williams-Hawkings Solvers for Airframe Noise Applications," AIAA Paper 2002-2580, 8th AIAA/CEAS Aeroacoustics Conference, Breckenridge, CO, June 17-19, 2002.

${ }^{10}$ Crighton, D. G., Dowling, A. P., Ffowcs Williams, J. E., Heckl, M., and Leppington, F. G., Modern Methods in Analytical Acoustics, chap. 11, Springer-Verlag, London, 1992, pp. 334-342.

${ }^{11}$ Farassat, F., "Linear Acoustic Formulas for Calculation of Rotating Blade Noise," AIAA Journal, Vol. 19, No. 9, 1981, pp. $1122-1120$.

${ }^{12}$ Lockard, D. P., Khorrami, M. R., and Li, F., "Aeroacoustic analysis of a simplified landing gear," AIAA-03-3111, Presented at the 9th AIAA/CEAS Aeroacoustics Conference and Exhibit in Hilton Head, SC, 2003.

${ }^{13}$ Rumsey, C., Biedron, R., and Thomas, J., "CFL3D: Its History and Some Recent Applications,” TM 112861, NASA, May 1997, presented at the Godonov's Method for Gas Dynamics Symposium, Ann Arbor, MI.

${ }^{14}$ Krist, S. L., Biedron, R. T., and Rumsey, C., "NASA Langley Research Center: Aerodynamic and Acoustic Methods Branch," CFL3D User's Manual (Version 5), 1997.

${ }^{15}$ Li, F., Khorrami, M. R., and Malik, M. R., "Unsteady Simulations of a Landing-Gear Flow Field," AIAA Paper 2002-2411, 8th AIAA/CEAS Aeroacoustics Conference, Breckenridge, CO, June 17-19, 2002.

${ }^{16}$ Menter, F. R., “Zonal Two-equation k-omega Turbulence Models for Aerodynamic Flows,” AIAA-93-2906, 1993.

${ }^{17}$ Menter, F. R., “Two-equation Eddy-viscosity Turbulence Models for Engineering Applications,” AIAA Journal, Vol. 32, No. 8, 1994, pp. 15981605.

${ }^{18}$ Singer, B. A., Lockard, D. P., and Brentner, K. S., "Computational Aeroacoustic Analysis of Slat Trailing-Edge Flow," AIAA Journal, Vol. 38, No. 9, 2000, pp. 1558-1564.

${ }^{19}$ Khorrami, M. R., Singer, B. A., and Berkman, M. E., "Time-accurate Simulations and Acoustic Analysis of Slat Free Shear Layer," AIAA Journal, Vol. 40, No. 7, 2002, pp. 1284-1291.

${ }^{20}$ Spalart, P. R., "Strategies for Turbulence Modeling and Simulations," International Journal of Heat and Fluid Flow, Vol. 21, 2000, pp. $252-263$.

${ }^{21}$ Spalart, P. R., "Young Person's Guide to Detached-Eddy Simulation Grids," CR 211032, NASA, 2001.

${ }^{22}$ Lazos, B. S., "Surface Topology on the Wheels of a Generic Four-Wheel Landing Gear," AIAA Journal, Vol. 40, No. 14, 2002 , pp. $2402-2411$.

${ }^{23}$ Lazos, B. S., "Reynolds Stresses Around the Wheels of a Simplified Four-Wheel Landing Gear," AIAA Journal, Vol. 42, No. 1, 2004 , pp. 196-198.

${ }^{24}$ Jaeger, S. M., Burnside, N. J., Soderman, P. T., Horne, W. C., and James, K. D., "Microphone Array Assessment of an Isolated, 26\%-scale, High-fidelity Landing Gear," AIAA Paper 2002-2410, 8th AIAA/CEAS Aeroacoustics Conference, Breckenridge, CO, June 17-19, 2002.

${ }^{25}$ Singer, B. A., Lockard, D. P., Brentner, K. S., and Lilley, G. M., "Simulation of Acoustic Scattering from a Trailing Edge," Journal of Sound and Vibration, Vol. 230, No. 3, 2000, pp. 541-560. 